\title{
Dosimetric Accuracy Using the New Mathematical Tools for Inhomogeneous Denser Medium
}

\author{
M. Jahangir Alam ${ }^{1, ~ *}$, M. Ashrafur Rahman ${ }^{2}$, Khandoker Siddique-E. Rabbani ${ }^{3}$ \\ ${ }^{1}$ Clinical Oncology Department, Ahsania Mission Cancer \& General Hospital, Dhaka, Bangladesh \\ ${ }^{2}$ Department of Arts \& Sciences, AUST, Dhaka, Bangladesh \\ ${ }^{3}$ Department of Biomedical Physics \& Technology, University of Dhaka, Dhaka, Bangladesh
}

Email address:

jahangir.rmf@gmail.com (M. J. Alam)

${ }^{*}$ Corresponding author

\section{To cite this article:}

M. Jahangir Alam, M. Ashrafur Rahman, Khandoker Siddique-E. Rabbani. Dosimetric Accuracy Using the New Mathematical Tools for Inhomogeneous Denser Medium. International Journal of Clinical and Experimental Medical Sciences. Vol. 3, No. 2, 2017 , pp. $23-29$. doi: $10.11648 / j$. .jjcems.20170302.12

Received: April 6, 2017; Accepted: April 19, 2017; Published: May 19, 2017

\begin{abstract}
It is possible to obtain a quality assurance (QA) of the dosimetry within a short time by using the new mathematical tools for a water phantom where dose measurements were made at two points only for a few square field sizes of the linear accelerator beam. The human body is not homogeneous. Water phantom makes it possible to create inhomogeneous phantoms by introducing blocks within it at suitable position to simulate body organs that may affect the dosage significantly. Two low cost inhomogeneous phantoms were developed using cork sheets and acrylic blocks to simulate the effects of normal lungs and cancerous lungs respectively using finite geometry and layer geometry. Monte Carlo Simulation was performed for each of these phantoms and detailed vertical and horizontal dose measurements were carried out. Percentage Depth Dose (PDD) measurements performed for the two point formalisms fixed at $100 \mathrm{~cm}$ Source to Surface Distance for both the homogeneous and inhomogeneous mediums and were compared with the doses generated by a Treatment Planning System. The quality of the methodology has ascertained firstly for a homogeneous medium. The formulated formalism of Tissue phantom ratio (TPR) was employed for inhomogeneous media particularly for finite and layer geometry using scattering factors obtained initially from detailed depth dose measurements. TPR conversion factors from homogeneous to inhomogeneous geometry were determined. The scattering factor was determined as a ratio of the depth dose in inhomogeneous medium and homogeneous medium. The quality factors of TPR values of homogeneous to inhomogeneous TPR conversion factor were also calculated. For all cases, the present results gave values which agreed very well to either actually measured values or with values calculated using TPS and these were also less than the international standard of deviation of $5 \%$. The low cost inhomogeneous phantoms through modifications of the water phantom deliver better information on QA consuming less time than before and offering better QA than a detector array. The present work will have an impact on the quality assurance of dosimetry and safety of radiotherapy.
\end{abstract}

Keywords: $\mathrm{TPR}=$ Tissue Phantom Ratio, $\mathrm{TPR}(\mathrm{EQS}) \mathrm{H}=$ Equation Simulated TPR for Homogeneous, TPR(TPS)H = TPR from TPS in Homogeneous, TPR(EQS)I = Simulated TPR for Inhomogeneous, TPR(TPS)I = TPR from TPS in Inhomogeneous, TPR(EQG)I = Generated TPR in Inhomogeneous, TPR(TPG)I $=$ TPS Generated TPR in Inhomogeneous

\section{Introduction}

It is estimated that approximately $40 \%$ of all cancer patients undergo radiation therapy with curative or palliative intent. It is assumed that $25 \%$ of all the cancer cases can be cured either by radiotherapy alone or through various combinations of surgery, radiotherapy and chemotherapy [1, $2,3]$. The main objective of radiotherapy is to enhance the tumor control by delivering maximum dose to the target, while reducing dose to the normal tissues [4]. It is, generally, 
given with the external beams of photons or electron produced by a medical linear accelerator. Gamma radiation using ${ }^{60} \mathrm{Co}$ is being gradually replaced by LINACs in most places.

Quality Assurance (QA) of a radiotherapy system is vital for quality and hazard free treatment to patient. Wrongly given radiotherapy will do more harm than good to a patient. The QA has two aspects. One is overall QA done in details initially during commissioning of the system, and then periodically, typically once each year. This QA procedure may require weeks.

Tissue phantom ratio (TPR), one of the several radiation dosimetry quantities, is being used to describe the change in dose with depth in tissue [5-9]. TPR data measurement needs to setup the ionization chamber in different conditions in air and in water. The setup varies with depth for different field sizes. So the whole procedure is time consuming and has the possibility to loss the consistency of measurement. But it is easy to calculate the TPR value for any field size of different depths in water from the percentage depth dose (PDD) values measured at two points of any field size at any depth and has less chance to make mistake because the ion chamber placement was done once at the central axis of the beam [8].

The aim of the present work:

\subsection{TPR Measurement from Two Point's Technique}

Measurement of PDD at two points only in a homogeneous medium to calculate TPR values at all other points, thereby reducing the QA time for commissioning and periodic calibration of the system, and most importantly making QA of TPS for individual patients possible using a water phantom with the newly developed formula [8].

\subsection{Application of the New Technique}

Application of the analytical formalism for QA in i) an inhomogeneous water phantom with the inhomogeneity distributed vertically on one side of the beam (termed 'finite geometry here') and in ii) an inhomogeneous water phantom with the inhomogeneity distributed horizontally near the surface, termed layer geometry. The two inhomogeneities introduced to simulate a normal lung and a lung with cancer on the top surface.

To achieve the above objectives, initially a mathematical formulation based on which a whole QA procedure for a LINAC using a water phantom was performed first for a homogeneous medium for different field sizes of the beam. QA was also being attempted with TPS predicted dose distribution for each of the field sizes. Measurements made on two types of inhomogeneous phantoms (block of corks, polystyrene etc immersed in the water phantom at suitable locations). Two data sets were compared to find out the percentage of deviation and to find out whether the formulation gives a reasonable QA or not. For acceptability, IAEA-recommendation was the criteria used in the present work.

For improving the treatment quality of cancer and reduce the uncertainty of delivering dose, there is no alternative to maintaining the accuracy and quality assurance of radiotherapy treatment planning. In that sense, the present work will help to improve the present treatment modality and quality assurance of the radiation dose measurement.

\section{Materials and Methods}

To simulate finite lung tissue having a single mass, the same rectangular water phantom of size $40 \mathrm{~cm} \times 40 \mathrm{~cm} \times$ $40 \mathrm{~cm}$ was used. A block of cork of density $0.30 \mathrm{~g} / \mathrm{cc}$ and of size $8 \mathrm{~cm} \times 8 \mathrm{~cm} \times 8 \mathrm{~cm}$ considered to simulate the human lung. In this phantom, the cork was placed $4 \mathrm{~cm}$ below the top of the water surface, at a distance of $4 \mathrm{~cm}$ from the left wall inside the water phantom as shown in Figure 1.

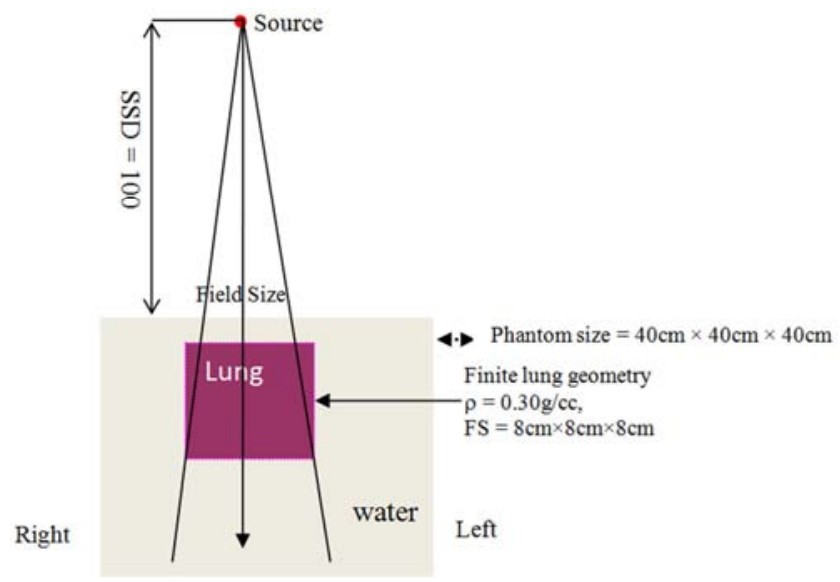

Figure 1. Schematic diagram of an in-homogeneity nested in finite. lung.

The ion chamber was placed $2.0 \mathrm{~cm}$ below the water surface along the central axis of the beam. The radiation of 6 MV photon beam was applied from above as shown in the cross sectional diagram in Figure1. The dose was measured along the central axis depth of the radiation field in the water phantom. Seven different field sizes from $5 \mathrm{~cm} \times 5 \mathrm{~cm}$ to 20 $\mathrm{cm} \times 20 \mathrm{~cm}$ were used. For depth dose measurements along the central axis in the phantom, a type 31006 ion chamber with $0.125 \mathrm{cc}$ volume was used together with an electrometer.

TPS, a conventional dose calculation system was used. A treatment plan made for in-homogenous phantom gave central axis depth distributions for different field sizes. Actual dose measurements were carried out in two dimensions. For each point, 3 readings were taken and the average was taken which is $\pm 1.5 \%$. The uncertainty of measurement was also determined. The treatment plan data correlated with the measured data to determine the efficacy of simple inhomogeneous phantom.

In order to get reliable results, the chamber was connected to the electrometer for 10 minutes and pre irradiated with 2 Gy, following the manufacturer's recommendations. For each point, the average of three readings was taken. The readings were corrected for temperature and pressure changes during measurements. The reference point for normalization of readings was at a depth of $5 \mathrm{~cm}$ on the central axis of the 
beam [9]. The photon beam direction was fixed along the zero angle of the gantry as well as the zero angle of the collimator. Two different treatment plans were developed for different field sizes for the inhomogeneous water phantom. The ion chamber was moved longitudinally from $2.0 \mathrm{~cm}$ to $20 \mathrm{~cm}$ with an increment of $0.5 \mathrm{~cm}$ between consecutive measurements. The field sizes of the beam were fixed at 100 $\mathrm{cm}$ SSD for all field sizes and depth-doses recorded in units of cGy/MU. For all the measurements, temperature, pressure and humidity should be kept at the same level, ideally. However, it was not possible since the measurements were carried out on different days at different times. Therefore, appropriate corrections using established factors were introduced to achieve the above objective within the cork, there is a reduction in dose and all the calculation methods that assume equilibrium over-predict the dose. Considering the average longitudinal range of an electron set in motion by $6 \mathrm{MV}$ X-rays to be $1.5 \mathrm{~cm}$ in water (i.e., $\mathrm{d}_{\max }$ ), this range is elongated to approximately $5.0 \mathrm{~cm}$ in cork with density of $0.30 \mathrm{~g} / \mathrm{cc}$. Therefore, electrons scattered to distance of approximately $5 \mathrm{~cm}$ laterally into the cork. Thus, irradiation of cork with a minimum field width equal to twice this lateral range (i.e., 6 to $10 \mathrm{~cm}$ ) is needed to maximize dose along the central axis of the beam.

To simulate lung tissue having an extensive tumour and possibility of using the new formation for inhomogeneous medium, an adequate phantom as well, layer geometry was used. For study, rectangular block of polystyrene sheet of density $1.06 \mathrm{~g} / \mathrm{cc}$ was used. Assumed that only a single fraction of radiation irradiated from zero angle of gantry and the phantom has to be designed for layer geometry accordingly. It used a rectangular piece of polystyrene of density $1.06 \mathrm{~g} / \mathrm{cc}$ and of size of $40 \mathrm{~cm}(\mathrm{l}) \times 40 \mathrm{~cm}(\mathrm{~b}) \times 1.5 \mathrm{~cm}$ (h) and the schematic diagram is shown in Figure 2

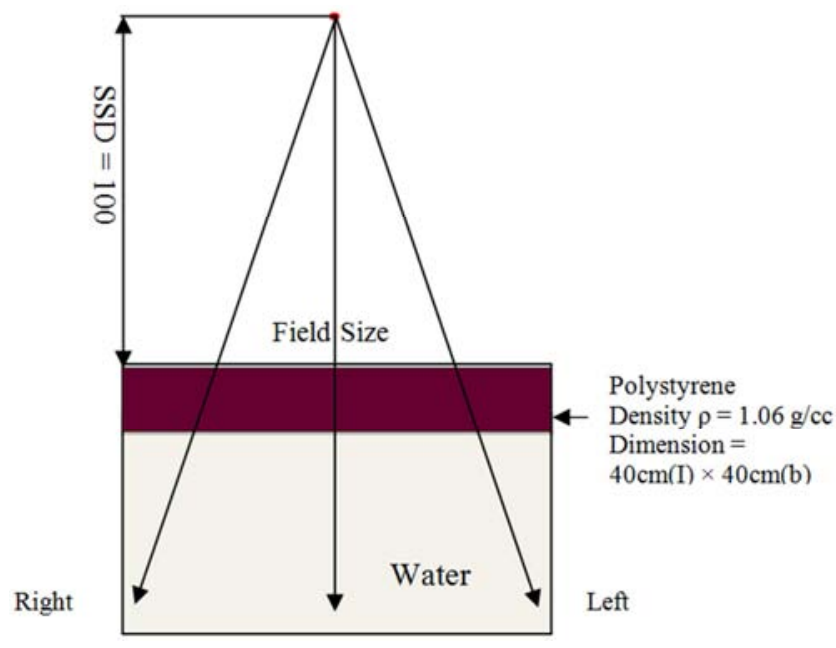

Figure 2. Schematic diagram of an in-homogeneity nested of Layer geometry.

Since the horizontal dimension of the phantom is $40 \mathrm{~cm} \times$ $40 \mathrm{~cm}$, the polystyrene piece covered the whole horizontal area of the phantom. The polystyrene block fixed inside the water phantom such that the bottom of the polystyrene block remains at a depth of $1.5 \mathrm{~cm}$ from the surface of water. The water phantom had a dimension of $40 \mathrm{~cm} \times 40 \mathrm{~cm} \times 40 \mathrm{~cm}$. The radiation was applied from above with field sizes of $5 \mathrm{~cm}$ $\times 5 \mathrm{~cm}, 7 \mathrm{~cm} \times 7 \mathrm{~cm}, 9 \mathrm{~cm} \times 9 \mathrm{~cm}, 11 \mathrm{~cm} \times 11 \mathrm{~cm}, 17 \mathrm{~cm} \times 17 \mathrm{~cm}$ and $20 \mathrm{~cm} \times 20 \mathrm{~cm}$. The reference point for the normalization of readings was at a depth of $1.5 \mathrm{~cm}$ (depth of maximum dose) on the central axis of the beam. Dose measurements and TPS calculations were performed for photon beam energy of $6 \mathrm{MV}$ for different field sizes.

By comparing the results of the TPS calculations with the water phantom measurements, the accuracy of measured depth dose and TPS generated depth dose for both the homogenous and inhomogeneous phantoms was checked. A treatment plan made for the above in-homogenous phantom for an SSD of $100 \mathrm{~cm}$ which gave central axis depth distributions for different field sizes. The scattering factor was calculated as the ratio of measured depth dose at a point in an inhomogeneous medium and homogeneous medium of the corresponding points. These factors required to calculate the TPR values of inhomogeneous medium using the newly formulated formalism [8].

For the study of layer geometry through the phantom measurements, the depth dose data for different field sizes were used. The geometries of the phantom and the field sizes were provided for the TPS calculations. For the phantom measurements, $0.6 \mathrm{~cm}$ increment was considered from $2 \mathrm{~cm}$ to $20 \mathrm{~cm}$ in longitudinal (vertical) direction. Same condition was considered for TPS simulation as well. Both the obtained doses were corrected due to temperature and pressure and plotted against corresponding depth $(\mathrm{cm})$.

The newly formulated formalism was applied to obtain TPR values for inhomogeneous layer geometry medium through modification based on scattering factor from layer geometry medium. The present study described the conversion of the measured data values into a comprehensive and consistent data set by the simulated formula that gives the TPR from PDD with depth as a function of field sizes. Firstly, depth dose for different field sizes measured in both the homogeneous $[\mathrm{DD}(\mathrm{M}) \mathrm{H}]$ and inhomogeneous [DD(M)I]. The scattering factors for different field sizes corresponding depths calculated the ratio of depth doses (inhomogeneous/homogeneous). TPR values were simulated for homogeneous medium [TPR(EQS)H] and for inhomogeneous medium [TPR(TPS)H] using two depths, taken from the $\mathrm{DD}(\mathrm{M}) \mathrm{H}$ obtained. Then, the scattering factor was introduced into the TPR(EQS)H together with SSD correction factor to achieve TPR(EQG)I for the inhomogeneous layer geometry medium (EQG calls for 'equation generated'). Secondly, $\mathrm{DD}(\mathrm{M}) \mathrm{I}$ for the inhomogeneous geometry medium was introduced to obtained another set of TPR values for the inhomogeneous medium which termed as TPR(EQS)I (EQS mentioned for equation simulated). Then both the TPR (EQG) I and TPR (EQS) I were compared to study the percentage difference between the two sets of results. 


\section{Results}

The comparative study of measured depth dose $\mathrm{DD}(\mathrm{M}) \mathrm{H}$ and TPS generated depth dose DD(TPS)H in homogeneous medium are shown in Figure 3 for different field sizes.

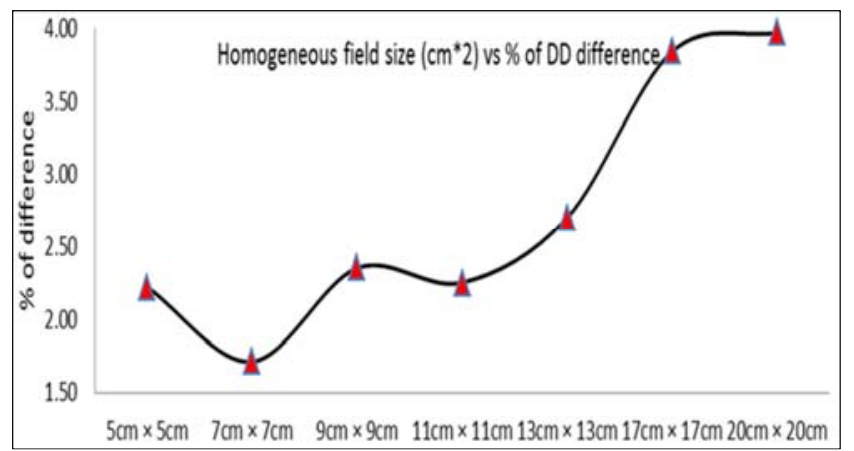

Figure 3. Comparison of measured depth dose and TPS generated depth dose for homogeneous medium.

Similar comparative study of measured depth dose DD(M)I and TPS generated depth dose DD(TPS)I for the specific inhomogeneous finite geometry medium are shown in Figure 4 for different field sizes.

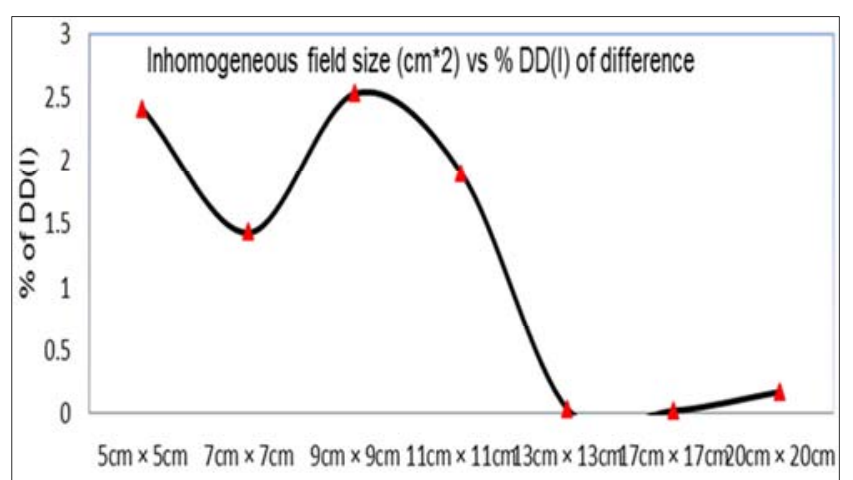

Figure 4. Comparison of measured depth dose and TPS generated depth dose for inhomogeneous medium.

It is seen that the corresponding values both the cases agree very well. The range of percentage difference between $\mathrm{DD}(\mathrm{M}) \mathrm{H}$ and $\mathrm{DD}(\mathrm{TPS}) \mathrm{H}$ are given in Table 1 while that between DD(M)I and DD(TPS)I are given in Table 2.

Table 1. Range of percentage of difference between DD (M) H and DD (TPS) $H$.

\begin{tabular}{lllllll}
\hline $\mathbf{5 c m} \times \mathbf{5} \mathbf{c m}$ & $\mathbf{7} \mathbf{c m} \times \mathbf{7} \mathbf{c m}$ & $\mathbf{9 c m} \times \mathbf{9 c m}$ & $\mathbf{1 1} \mathbf{c m} \times \mathbf{1 1} \mathbf{c m}$ & $\mathbf{1 3} \mathbf{c m} \times \mathbf{1 3} \mathbf{c m}$ & $\mathbf{1 7} \mathbf{c m} \times \mathbf{1 7} \mathbf{c m}$ & $\mathbf{2 0 c m} \times \mathbf{2 0} \mathbf{c m}$ \\
\hline $\pm 2.22 \%$ & $\pm 1.71 \%$ & $\pm 2.35 \%$ & $\pm 2.25 \%$ & $\pm 2.70 \%$ & $\pm 3.84 \%$ & $\pm 3.97 \%$ \\
\hline
\end{tabular}

Table 2. Range of percentage of difference between DD (M)I and DD(TPS)I.

\begin{tabular}{lllllll}
\hline $\mathbf{5 c m} \times \mathbf{5 c m}$ & $\mathbf{7} \mathbf{c m} \times \mathbf{7} \mathbf{c m}$ & $\mathbf{9 c m} \times \mathbf{9 c m}$ & $\mathbf{1 1} \mathbf{c m} \times \mathbf{1 1} \mathbf{c m}$ & $\mathbf{1 3 c m} \times \mathbf{1 3} \mathbf{c m}$ & $\mathbf{1 7} \mathbf{c m} \times \mathbf{1 7} \mathbf{c m}$ & $\mathbf{2 0} \mathbf{c m} \times \mathbf{2 0} \mathbf{c m}$ \\
\hline $\pm 2.41 \%$ & $\pm 1.43 \%$ & $\pm 2.53 \%$ & $\pm 1.90 \%$ & $\pm 0.03 \%$ & $\pm 0.02 \%$ & $\pm 0.17 \%$ \\
\hline
\end{tabular}

All the difference values are within $3.97 \%$ and $2.53 \%$ in Table 1 and Table 2 respectively which is less than the range of $\pm 5 \%$ accepted universally [10.11]. The comparative study of TPR (EQS) H (measured) and TPR (TPS) H (TPS generated) in homogeneous medium are shown in Figure 5 for different field sizes.

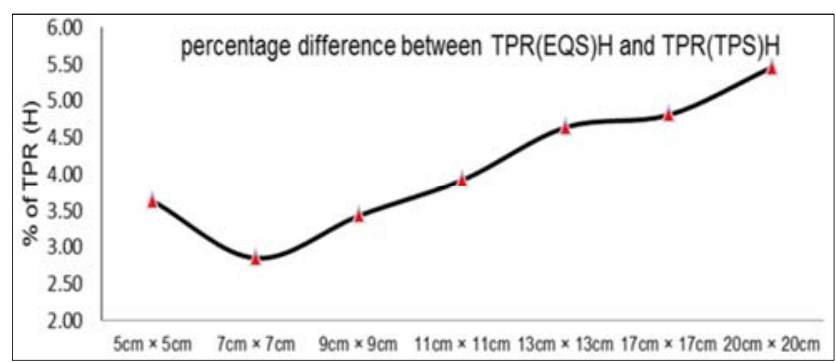

Figure 5. Comparison of measured TPR (EQS) and TPR (TPS generated) for homogeneous.

It is seen that the corresponding values agree very well. Similar comparative study of equation simulated TPR(EQS)I and TPS generated TPR(TPS)I for the specific inhomogeneous medium are shown in Figure 6 for different field sizes.

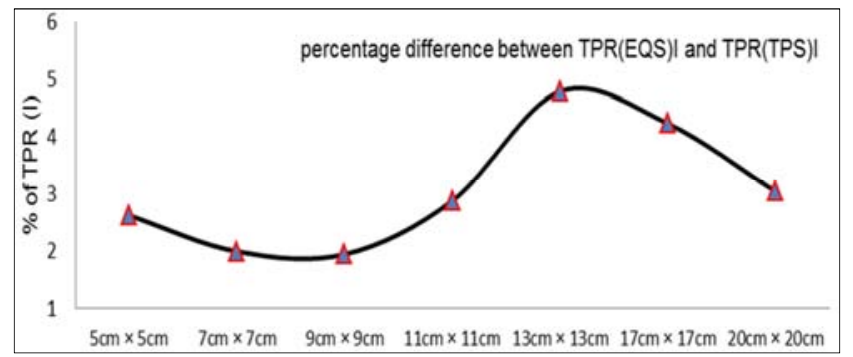

Figure 6. Comparison of equation simulated TPR (EQS) and TPS generated TPR for different field sizes of inhomogeneous medium.

It is seen that the corresponding values are consistent very well. The range of percentage difference between TPR(EQS)H and TPR(TPS)H and that between TPR(TEQS)I and TPR(TPS)I are given in Table 3 and in Table 4 respectively.

Table 3. Range of percentage of difference between TPR(EQS)H and TPR(TPS)H.

\begin{tabular}{|c|c|c|c|c|c|c|}
\hline $5 \mathrm{~cm} \times 5 \mathrm{~cm}$ & $7 \mathrm{~cm} \times 7 \mathrm{~cm}$ & $9 \mathrm{~cm} \times 9 \mathrm{~cm}$ & $11 \mathrm{~cm} \times 11 \mathrm{~cm}$ & $13 \mathrm{~cm} \times 13 \mathrm{~cm}$ & $17 \mathrm{~cm} \times 17 \mathrm{~cm}$ & $20 \mathrm{~cm} \times 20 \mathrm{~cm}$ \\
\hline $\pm 3.63 \%$ & $\pm 2.85 \%$ & $\pm 3.43 \%$ & $\pm 3.92 \%$ & $\pm 4.64 \%$ & $\pm 4.81 \%$ & $\pm 4.46 \%$ \\
\hline
\end{tabular}


Table 4. Range of percentage difference between TPR (EQS) I and TPR (TPS)I.

\begin{tabular}{|c|c|c|c|c|c|c|}
\hline $5 \mathrm{~cm} \times 5 \mathrm{~cm}$ & $7 \mathrm{~cm} \times 7 \mathrm{~cm}$ & $9 \mathrm{~cm} \times 9 \mathrm{~cm}$ & $11 \mathrm{~cm} \times 11 \mathrm{~cm}$ & $13 \mathrm{~cm} \times 13 \mathrm{~cm}$ & $17 \mathrm{~cm} \times 17 \mathrm{~cm}$ & $20 \mathrm{~cm} \times 20 \mathrm{~cm}$ \\
\hline $\pm 2.62 \%$ & $\pm 1.99 \%$ & $\pm 1.94 \%$ & $\pm 2.88 \%$ & $\pm 4.79 \%$ & $\pm 4.23 \%$ & $\pm 3.04 \%$ \\
\hline
\end{tabular}

The percentage differences of most of TPRs are less than 5 except one in Table 3. All the percentage differences are less than 4.79 in Table 4, which are within the range of $\pm 5 \%$ and showed a good agreement between equation simulation and TPS calculation.

Tissue phantom ratio calculated for homogeneous and inhomogeneous geometry medium by using the newly formulated formalism [8] for measured and TPS depth dose data. For quality assurance of measured and TPS generated data, TPR conversion factor determined from the equation simulated TPRs for a specific inhomogeneity for different fields and similarly TPR conversion factor also determined from TPS simulated TPRs for a specific inhomogeneity for different field sizes. The TPR conversion factor for $\mathrm{TPR}(\mathrm{EQS})_{\mathrm{H}-\mathrm{I}}$ and TPR(TPS $)_{\mathrm{H}-\mathrm{I}}$ from homogeneous to inhomogeneous geometry varies from 0.97 to 1.02 and 0.95 to 1.05 respectively. The percentages of differences between two TPRs values are maxima of $2.86 \%$, which are within the range accepted universally. This shows the good agreement of quality assurance between the two TPRs.

The quality factor TRP(EQG)H-I calculated from TPR(EQS)H and scattering factor. Both the TPR(EQG)I and TPR(EQS)I compared to study the percentage difference between the two sets of results. The percentage difference values are less than 4.5. Similarly, the quality factor of TPS simulated TPR(TPG)H-I and calculated the percentage difference of TPS simulated TPR(TPG)H-I and TPR(TPS)H-I. The percentage differences are maxima of \pm 1 which show the good agreement with equation simulation and TPS calculation.

The TPR conversion factors between TPR(EQS)H and TPR(EQS)H from equation simulation for a specific homogeneity layer geometry medium for different field sizes given in Table 5, and the values vary from 0.56 to 4.69 .

Table 5. Range of percentage of difference between measured TPR values of $T P R(E Q S) H$ and TPS generated TPR values of TPR(TPS)H.

\begin{tabular}{llll}
\hline $7 \mathrm{~cm} \times 7 \mathrm{~cm}$ & $9 \mathrm{~cm} \times \mathbf{9 c m}$ & $\mathbf{1 1} \mathrm{cm} \times 11 \mathrm{~cm}$ & $17 \mathrm{~cm} \times \mathbf{1 7} \mathrm{cm}$ \\
\hline $\pm 4.69 \%$ & $\pm 0.56 \%$ & $\pm 0.64 \%$ & $\pm 2.22 \%$ \\
\hline
\end{tabular}

The percentage difference between TPR(EQS)H and TPR(TPS)H for homogeneous medium is also shown graphically in Figure 7.

Similarly the TPR conversion factor between TPR(EQS)H and TPR(TPS)I was evaluated from TPS generated data and the values vary from 0.17 to 4.9 given in Table 6 .

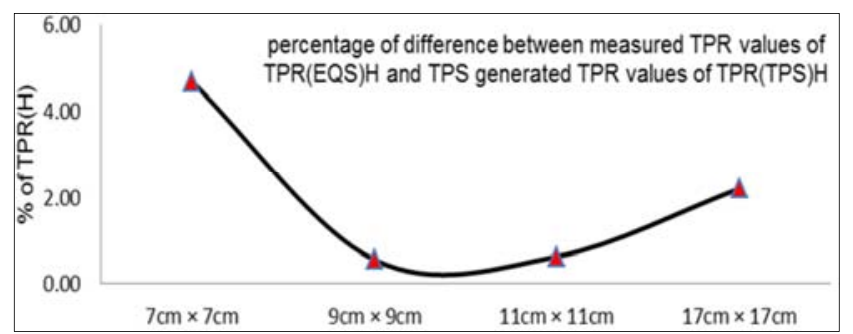

Figure 7. Percentage difference between TPR(EQS) and TPR(TPS) for homogeneous medium.

Table 6. Range of percentage of difference between measured TPR values of TPR(EQS)H and TPS generated TPR values of TPR(TPS)I.

\begin{tabular}{lllllll}
\hline $\mathbf{5 c m} \times \mathbf{5 c m}$ & $\mathbf{7 c m} \times \mathbf{7 c m}$ & $\mathbf{9 c m} \times \mathbf{9 c m}$ & $\mathbf{1 1} \mathbf{c m} \times \mathbf{1 1} \mathbf{c m}$ & $\mathbf{1 3} \mathbf{c m} \times \mathbf{1 3} \mathbf{c m}$ & $\mathbf{1 7} \mathbf{c m} \times \mathbf{1 7} \mathbf{c m}$ & $\mathbf{2 0 c m} \times \mathbf{2 0} \mathrm{cm}$ \\
\hline $\pm 1.7 \%$ & $\pm 1.77 \%$ & $\pm 2.2 \%$ & $\pm 3.1 \%$ & $\pm 4.1 \%$ & $\pm 4.7 \%$ & $\pm 4.9 \%$ \\
\hline
\end{tabular}

The percentage difference of TPR(EQS)I and TPR(TPS)I for inhomogeneous medium are also shown graphically in Figure 8.

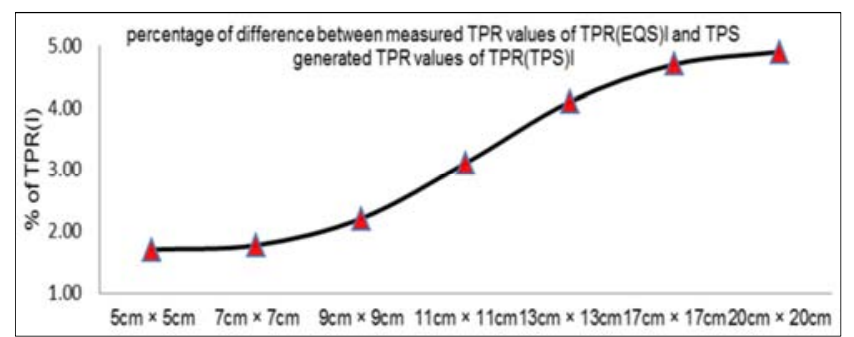

Figure 8. Percentage difference between $T P R(E Q S)$ and TPR(TPS) for inhomogeneous medium.

The percentages of two TPRs values are maxima of 4.9 which are within the range of acceptability universally. This shows a good agreement of quality assurance in inhomogeneous medium. From the percentage difference of
TPR conversion factor between TPR(EQS $)_{\mathrm{H}-\mathrm{I}}$ and TPR(TPS $)_{\mathrm{H}-\mathrm{I}}$ values, it is seen that all the difference values are less than $4.85 \%$. The quality factor of equation TPR(EQG)H-I generated for the specific inhomogeneity layer geometry medium based on the equation. The TRP(EQG)H-I has calculated from the TPR(EQS)H and the scattering factor. Both the TPR(EQG)I and TPR(EQS)I were compared to study the percentage difference between the two sets of results. The percentage difference values are less than 3. Similarly, the quality factor of TPS calculated from the percentage difference of TPS simulated TPR(TPG)H-I and TPR(TPS)H-I. The percentage difference is maximum \pm 1.5 which shows the good agreement with the equation simulation and TPS calculation.

The Validation of New formalism is given in tabulated form in Table 7 . 
Table 7. Validation of the new formalism.

\begin{tabular}{|c|c|c|c|c|c|c|}
\hline \multirow[b]{2}{*}{ Parameters(using new formalism) } & \multicolumn{2}{|c|}{ Homogeneous medium } & \multicolumn{4}{|c|}{ Inhomogeneous finite lung geometry medium } \\
\hline & TPR(EQS)H & TPR(EQS)H & TPR(EQS)I & TPR(EQS)H-I & TPR(EQS)I & TPR(TPG)I \\
\hline Standard parameter using ( TPS ) & TPR(TPS)H & TPR(TPS)H & TPR(TPS)I & TPR (TPS)H-I & TPR(EQG)I & TPR(TPS)I \\
\hline Maximum percentage difference & $\pm 4.81 \%$ & $\pm 4.69 \%$ & $\begin{array}{l} \pm 4.79 \% \\
\pm 4.9 \%\end{array}$ & $\begin{array}{l} \pm 2.86 \% \\
\pm 4.85 \%\end{array}$ & $\begin{array}{l} \pm 4.5 \% \\
\pm 3.0 \%\end{array}$ & $\begin{array}{l} \pm 1.0 \% \\
\pm 1.5 \%\end{array}$ \\
\hline
\end{tabular}

The first row is the parameters generated using the new formalism. The second row lists the parameters which are used as standards to compare the above parameters. These comparisons performed for different field sizes. The third row gives the maximum difference or ratio, as appropriate, obtained for all the field sizes for each parameter.

Since the measurements performed at three different times with one in a complete different set-up, essentially the same measurement was taken three times for the homogeneous medium. The maximum difference was $5.6 \%$ in only one case of $1 \mathrm{~cm} \times 1 \mathrm{~cm}$ field size which have counted out in this table. At such small field size, the error may be larger because of the finite dimension of the ion chamber due to an outer diameter of $1.2 \mathrm{~cm}$, the sensitive volume being $0.125 \mathrm{cc}$.

\section{Discussions}

Though Detector Array (DA) gives a fast output but it has a limitation that it does not measure the actual dose to be received by the patient, besides being very expensive. This device can predict doses for only homogeneous fields, while in real life we encounter inhomogeneous fields due to scattering from various organs within the human body. On the other hand, the water phantom has the advantage that it measures almost the real dose received by a patient. This measurement includes the radiation scattered from the water body which is similar to that occurring in a real human body. It is also much less expensive than a detector array. The human body is not homogeneous. This inhomogeneity may be simulated to some extent at low cost by introducing blocks of different materials within the water body at suitable positions. Although human body phantoms with DA's are being introduced recently, the cost is very high, and impractical for a Third World situation.

The QA using water phantom performed through actual measurement of dose distributions. The QA device placed at the iso-centre of the linear accelerator to measure the dose of beam profile and PDD at a single distance for individual field sizes and the dose to be received by the patient is predicted through mathematical simulations. The present work based on the newly developed mathematical formulation [8] allows QA through measurements at two points only in a water phantom in SSD which makes it simple and less time consuming and has less chance to make mistake because the ion chamber placement was done once at the central axis of the beam.

The developed method can also reduce the human error in the QA performed in commissioning process of the radiotherapy system and at periodic intervals. The whole QA can be done in one session of an hour during which variation in temperature and pressure is negligible. Besides, human error expected in a prolonged measurement will be reduced through this short measurement procedure. Elaborate measurements performed to determine the suitability of using the new formulation based on two-point measurements in a water phantom. The measurements were performed in the phantom of size $40 \mathrm{~cm} \times 40 \mathrm{~cm} \times 40 \mathrm{~cm}$ using small volume ion chamber (one with $0.3 \mathrm{cc}$, and the other with $0.125 \mathrm{cc}$ ). A smaller chamber may give more accurate results, but was not available. The absorbed doses were measured at various depths for different field sizes from $5 \mathrm{~cm} \times 5 \mathrm{~cm}$ to $30 \mathrm{~cm} \times$ $30 \mathrm{~cm}$. For QA, different combinations of measured and simulated TPR values were investigated in several types of media. These included a homogeneous phantom and phantoms with inhomogeneity in the forms called 'finite geometry' and 'layer geometry' respectively to simulate normal lungs and a lung with cancer at the top layers respectively. Again different materials, i) water \& cork phantom and ii) polystyrene $\&$ water phantom were used to introduce inhomogeneity in the water phantom. The cork with a density of $0.3 \mathrm{~g} / \mathrm{cc}$ was used to simulate a low density region within the tissue and may represent normal lungs. On the other hand, a cancerous lung will have a tissue density almost equal to the other body tissues or even slightly higher. A polystyrene block with a density of $1.06 \mathrm{~g} / \mathrm{cc}$ was used for this purpose. Another phantom was designed and studied which involved a polystyrene block in the water phantom with a hole in its centre. This could be useful in real life situations in certain applications where a void is present within a mass of higher density.

For all cases, the new formalism gave values which agreed very well to either actually measured values or with values calculated using TPS, which took as standards in the respective comparisons. In all these cases, the difference from the respective standards was less than the globally agreed deviation of 5\%. However, it was found that for field sizes of $10 \times 10 \mathrm{~cm}^{2}$ and above, the deviation from TPS data was less than $2.5 \%$ which was very good according to the international standard. For smaller fields, the deviation was more than $2.5 \%$. This slightly higher deviation at smaller fields may be due to the inaccuracy in dose measurements in the build-up region because of volume of the ionization chamber. The other reason may be due to the difficulties in modeling of the electron contamination of the photon beam.

\section{Conclusions}

To improve the treatment quality of cancer and to reduce the uncertainty of delivering dose, there is no alternative to maintaining the accuracy and quality assurance of 
radiotherapy treatment planning.

It is found that the corresponding values of measured depth dose of different field sizes for both the mediums and TPS generated depth dose of different field sizes shown in Figure 3 and in Figure 4 respectively agree very well. It is also found that the range of percentage difference between them given in Table 1 and Table 2 are within 3.97 and 2.53 respectively which are less than the range accepted universally $[10,11]$.

Similarly for the other cases, the formalism gave values which agreed very well to either actually measured values or with values calculated using TPS, which took as standards in the respective comparisons of the corresponding values. However, it is found that for field sizes of $10 \times 10 \mathrm{~cm}^{2}$ and above, the deviation from TPS data was less than $2.5 \%$ which is good according to the international standard. The slightly higher deviation of that value for smaller fields may be due to the inaccuracy in dose measurements in the build-up region because of volume of the ionization chamber.

Calculating TPR value for any field size of different depths in water from the PDD values measured at two points of any field size at any depth and the placement of ion chamber at the central axis of the beam once reduces the quality assurance (QA) time for commissioning and periodic calibration of the system. The development of low cost inhomogeneous phantoms through modifications of the water phantom deliver better information on QA consuming less time than before, and offering better QA than a detector array. The present work will help to improve the radiotherapy modality and contributes a simple method for quality assurance and dose distribution for radiotherapy using LINACs. Therefore, it makes a practical proposition to perform QA of TPS for each patient using a water phantom.

\section{Acknowledgement}

The authors would like to acknowledge the Professor Guenther Hartmann, German Cancer Research Institute, Heidelberg, Germany for valuable suggestions regarding the present work. We also acknowledge the President, Dhaka Ahsania Mission \& Managing Director, Ahsania Mission Cancer \& General Hospital, Uttara, Dhaka, Bangladesh for giving opportunity to the authors to research there and thanks to all technical \& Clinical staffs of the Clinical Oncology Department for cooperation.

\section{References}

[1] The Physics of Radiology, Harold Elford Johns \& John Robert Cunningham, Third edition, Fifth printing, 1978.

[2] Sontag MR and Cunningham JR., Corrections to absorbed dose calculations for tissue in-homogeneities, Med. Phys, 1977; 4: 431-436.

[3] Woo MK and Cunningham JR, The validity of the density scaling method in primary electron transport for electron and photon beams, Med. Phys, 1990; 17: 187-194.

[4] Khan M. I, Runqing Jiang, Alexander Rehman Kiciak, Jalil ur, Muhammad Afzal, James C. L. Chow' Dosimetric and radiobiological characterizations of prostate intensitymodulated radiotherapy and volumetric-modulated arc therapy. J. Med. Phys, 2016;41:162-168.

[5] Purdy J. A. Relationship between tissue-phantom ratio and percentage depth dose. Med phys, 1977; 4: 66-7.

[6] Rahman M. A. and Jahangir Alam M.' Analysis of Tissue Phantom Ratio of the Megavoltage Photon Beams' Malays. j. med. biol. Res. 2016; 3: 61-68.

[7] Rahman M. A., Jahangir Alam M., Akhtaruzzaman M. 'Characteristics Analysis of High Energy External Radiotherapy Beams in Water' Malays. j. med. biol. Res. 2016; 3, 51-60.

[8] Jahangir Alam M. et al. A. modified formula for defining tissue phantom ratio of photon beams. Bangladesh Med. Coun Bull 2007; 33, 92-97.

[9] Jari Viitanen, Development and evaluation of a Dose Planning system for radiation therapy, Espoo 1989.

[10] Technical Report Series no.398, Absorbed Dose Determination in External Beam Radiotherapy. An International Code of Practice for Dosimetry Based on Standards of Absorbed Dose to Water, 2000.

[11] Technical Reports Series No. 430: Commissioning and Quality Assurance of Computerized Planning Systems for Radiation Treatment of Cancer, International Atomic Energy Agency, Vienna, 2004. 